\title{
Metastatic Biliary Stricture with "Beaded” Appearance from Cervical Adenocarcinoma
}

\author{
Soon Liang Lee ${ }^{1} \odot$, Jasminder Sidhu², Chiun Yann $\mathrm{Ng}^{3}$
}

\begin{abstract}
Cervical adenocarcinoma accounts for $25 \%$ of invasive cervical cancer which frequently metastasize distantly to the lungs, liver, bone, and brain. Metastases to the common bile duct from cervical cancer are exceedingly rare with few reported cases in the literature. Diagnosis of bile duct metastases from cervical cancer can be established with endoscopic ultrasound-guided fine-needle aspiration cytology, biliary cytobrushing, or direct cholangioscopy with biopsy, and this would guide further therapies such as endoscopic biliary drainage and systemic chemotherapy. We hereby present a rare case of obstructive jaundice from metastatic biliary stricture with "beaded" appearance in a patient with cervical adenocarcinoma.
\end{abstract}

Keywords: Biliary stricture, Cervical adenocarcinoma, Endoscopic retrograde cholangiopancreatography, Endoscopic ultrasound. Euroasian Journal of Hepato-Gastroenterology (2021): 10.5005/jp-journals-10018-1350

\section{INTRODUCTION}

Cervical adenocarcinoma accounts for $25 \%$ of invasive cervical cancer which frequently metastasize distantly to the lungs, liver, bone, and brain. Metastatic cervical cancer has dismal prognosis with five-year survival rate of $17.2 \%$. $^{1}$ The possible etiologies of obstructive jaundice in metastatic cervical cancer are liver metastases, extrinsic bile duct compression from porta hepatis lymphadenopathy, pancreatic and periampullary metastases. Metastases to bile duct from cervical cancer are extremely rare with few cases reported in the literature. ${ }^{2,3}$ We present a rare case of obstructive jaundice from metastatic biliary stricture with "beaded" appearance in a patient with cervical adenocarcinoma.

\section{Case Description}

A 60-year-old lady with history of cervical adenocarcinoma presented with jaundice, epigastric pain, fever, and constitutional symptoms for 2 weeks. She had total abdominal hysterectomy with bilateral salpingo-oophorectomy 3 years ago with chemoradiation therapy and was in complete remission. Physical examination revealed icteric sclera with mild epigastric tenderness. Laboratory investigation showed total bilirubin of $10.6 \mathrm{mg} / \mathrm{dL}$, alkaline phosphatase of 521 units/L, elevated serum CA-125 of 1194 units $/ \mathrm{mL}$, and normal CA-19-9 level of less than 2 units $/ \mathrm{mL}$. Computed tomography (CT) of the abdomen showed "beaded" appearance of common bile duct (CBD) with proximal CBD stricture and upstream bilateral proximal intrahepatic ducts dilatation (Fig. 1A). Endoscopic ultrasound (EUS) revealed irregular thickening of CBD wall causing stricture and "beaded" CBD appearance (Fig. 1B). EUS-guided fine-needle aspiration cytology (FNAC) was performed on the CBD stricture, and cellblock examination revealed poorly differentiated malignant cells with immunohistochemistry tests revealing positive stain for $\mathrm{ck} 7$ (Fig. 1C) and p16 (Fig. 1D), confirming metastatic deposits of cervical adenocarcinoma. Endoscopic retrograde cholangiopancreatography (ERCP) confirmed "beaded" CBD appearance locating in between the proximal and distal CBD stricture with dilated intrahepatic ducts (Fig. 2A). A single pigtail pancreatic duct plastic stent $4 \mathrm{fr} 5 \mathrm{~cm}$ was inserted to aid CBD
${ }^{1-3}$ Department of Medicine, Gastroenterology and Hepatology Unit, Kuala Lumpur General Hospital, Kuala Lumpur, Malaysia

Corresponding Author: Soon Liang Lee, Department of Medicine, Gastroenterology and Hepatology Unit, Kuala Lumpur General Hospital, Kuala Lumpur, Malaysia, e-mail: soonliang13@gmail.com

How to cite this article: Lee SL, Sidhu J, Ng CY. Metastatic Biliary Stricture with "Beaded" Appearance from Cervical Adenocarcinoma. Euroasian J Hepato-Gastroenterol 2021;11(2):97-99.

Source of support: Nil

Conflict of interest: None

cannulation, and a plastic stent $10 \mathrm{fr} 9 \mathrm{~cm}$ was inserted for biliary drainage (Fig. 2B) while waiting for final cytopathology report. She however succumbed to pulmonary embolism a week later.

\section{Discussion}

"Beaded" appearance typically seen in primary sclerosing cholangitis (PSC) occurs due to presence of multifocal strictures alternating with normal caliber ducts or dilated ducts. ${ }^{4}$ This case illustrates unique imaging findings of "beaded" bile duct appearance in a malignant CBD stricture arising from cervical adenocarcinoma. This case also highlights that metastatic biliary stricture may mimic PSC with dominant stricture or extrahepatic cholangiocarcinoma, and EUS-guided FNAC, biliary cytobrushing, or direct cholangioscopy with biopsy may help to confirm the underlying etiology. ${ }^{5}$ Clinical history, previous radiological assessment during initial diagnosis of cervical adenocarcinoma, and elevated Ca-125 with normal CA 19-9 made the diagnosis of PSC with extrahepatic cholangiocarcinoma highly improbable. Metastases to the biliary tract resulting in stricture are rare and usually originate primarily from cancer of the lung, breast, or colon. ${ }^{6}$ Although there were a few case reports of metastatic squamous cell carcinoma of the cervix with biliary stricture and obstructive jaundice, but to our knowledge, this is the first case report of biliary duct involvement from metastatic cervical adenocarcinoma. ${ }^{3}$ The preferred definitive management option for such case would be 

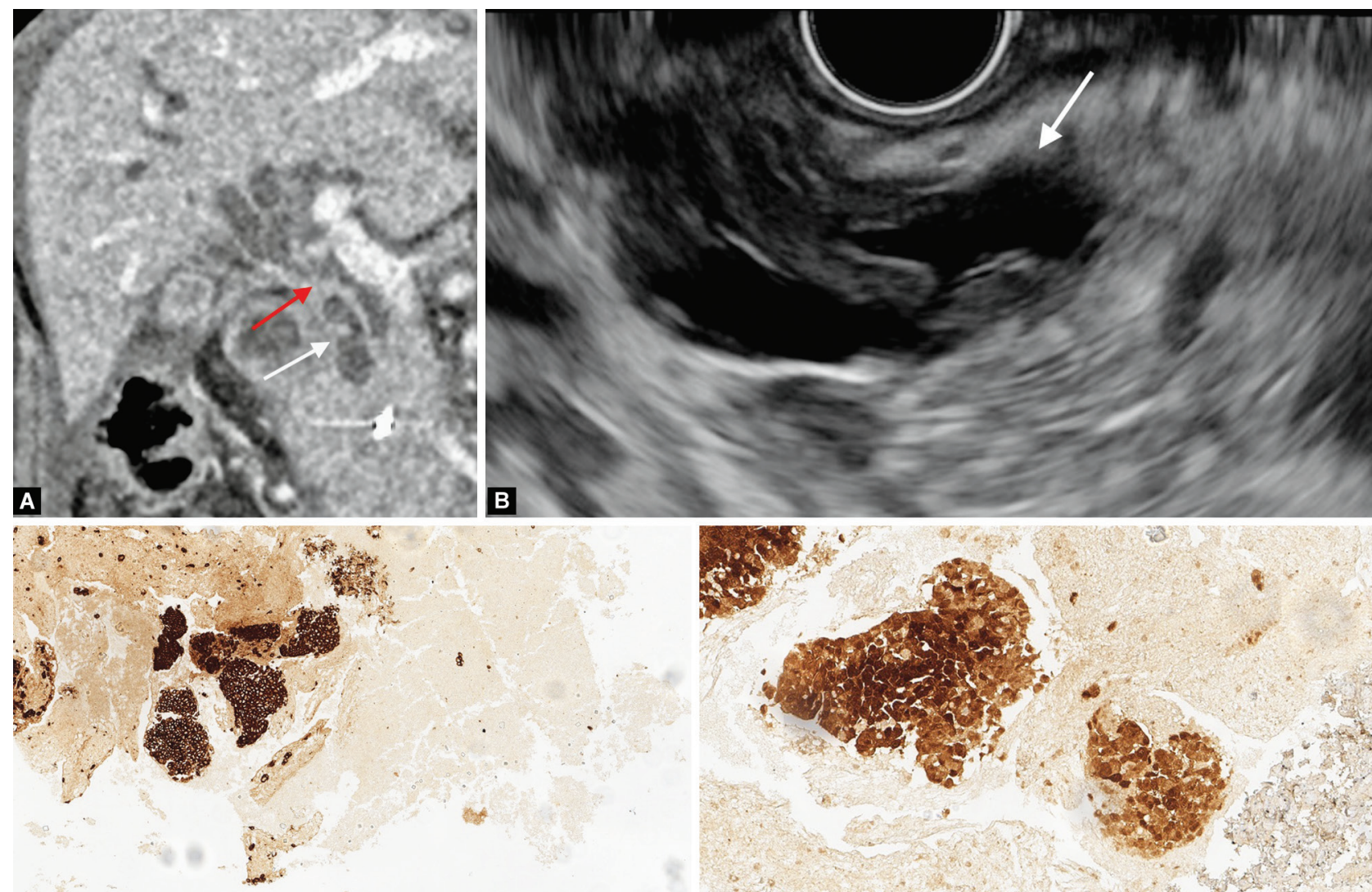

C.

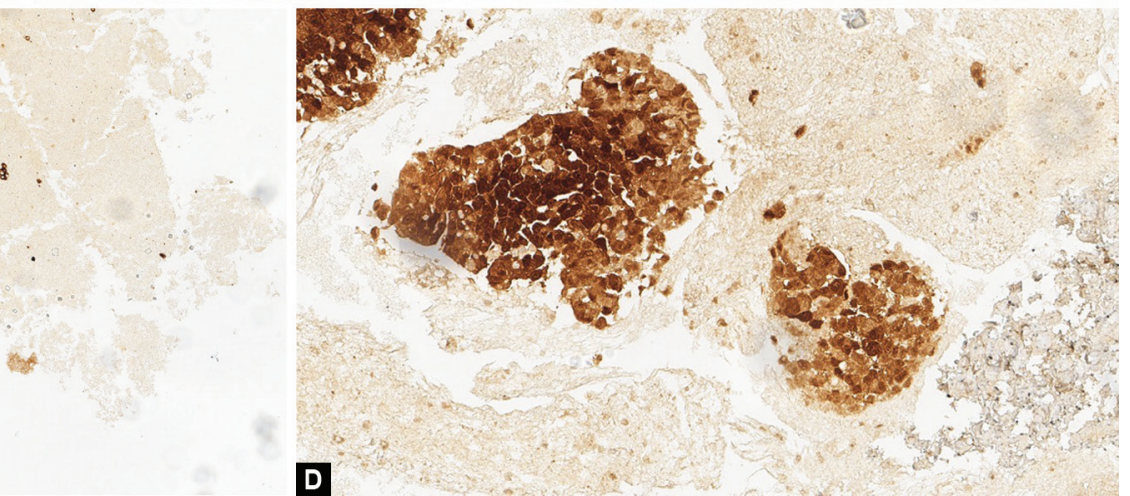

Figs 1A to D: (A) CT abdomen revealed a proximal CBD stricture (red arrow) with beading of bile duct (white arrow) and dilated proximal intrahepatic ducts and ascites; (B) EUS showed irregular CBD wall thickening with "beaded" appearance (white arrow). Poorly differentiated malignant cells with immunohistochemistry test positive for (C) ck7 and; (D) p16 stain confirming metastases from cervical adenocarcinoma
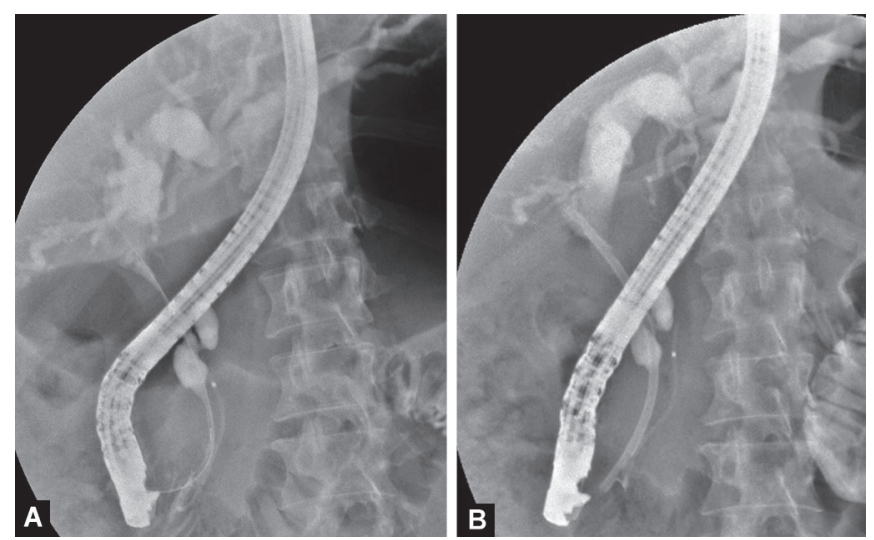

Figs $2 A$ and B: "Beaded" CBD locating in-between proximal and distal CBD stricture on contrast cholangiogram. (A) Pancreatic stent placement was performed to aid biliary cannulation; (B) Biliary plastic stent was inserted for biliary drainage after pancreatic stent placement

palliative endoscopic biliary drainage with self-expanding metallic stent as it has been proven to have better stent patency with less re-intervention rates. ${ }^{7}$

\section{CONCLUSION}

Bile duct metastases from cervical adenocarcinoma are rare and may mimic the cholangiographic features of PSC with dominant stricture or extrahepatic cholangiocarcinoma. EUS-guided FNAC, biliary cytobrushing, and direct cholangioscopy with biopsy are the modalities that can be considered in obtaining pathological diagnosis to determine further treatment.

\section{Clinical Significance}

Metastatic bile duct stricture should be considered in patient with cervical adenocarcinoma presenting with obstructive jaundice although it is an uncommon site of distant spread. Immunohistochemistry test performed on the tissue acquired from the stricture site with the modalities as explained above will help in establishing the underlying etiology.

\section{ACKnowledgment}

The authors would like to thank the Director General of Health Malaysia for his permission to publish this paper.

\section{OrCID}

Soon Liang Lee 이 https://orcid.org/0000-0003-0341-5394

\section{References}

1. Gardner AB, Charo LM, Mann AK, et al. Ovarian, uterine, and cervical cancer patients with distant metastases at diagnosis: most common locations and outcomes. Clin Exp Metastasis 2020;37(1):107-113. DOI 10.1007/s10585-019-10007-0. 
2. Nishimura $\mathrm{C}$, Naoe $\mathrm{H}$, Hashigo $\mathrm{S}$, et al. Pancreatic metastasis from mixed adenoneuroendocrine carcinoma of the uterine cervix: a case report. Case Rep Oncol 2013;6(2):256-262. DOI: 10.1159/000351308.

3. Levey JM. Endoscopic biliary drainage for metastatic squamous cell carcinoma of the cervix. Gastrointest Endosc 2000;51(1):97-99. DOI: 10.1016/s0016-5107(00)70401-9.

4. Vitellas KM, Keogan MT, Freed KS, et al. Radiologic manifestations of sclerosing cholangitis with emphasis on MR cholangiopancreatography. Radiographics 2000;20(4):959-975. DOI: 10.1148/ radiographics.20.4.g00jl04959.
5. Novikov A, Kowalski TE, Loren DE. Practical management of indeterminate biliary strictures. Gastrointest Endosc Clin N Am 2019;29(2):205-214. DOI: 10.1016/j.giec.2018.12.003.

6. Katabathina VS, Dasyam AK, Dasyam N, et al. Adult bile duct strictures: role of MR imaging and MR cholangiopancreatography in characterization. Radiographics 2014;34(3):565-586. DOI: 10.1148/ rg.343125211.

7. Wang CC, Yang TW, Sung WW, et al. Current endoscopic management of malignant biliary stricture. Medicina (Kaunas) 2020;56(3):114. DOI: 10.3390/medicina56030114. 